\title{
A Numerical Experiment in DLCQ: Microcausality, Continuum Limit and all that
}

\author{
Dipankar Chakrabarti, Asmita Mukherjee*, Rajen Kundu, A. Harindranath \\ Saha Institute of Nuclear Physics \\ 1/AF Bidhan Nagar, Calcutta, 700064 India
}

(February 2, 2000)

\begin{abstract}
Issues related with microcausality violation and continuum limit in the context of $(1+1)$ dimensional scalar field theory in discretized light-cone quantization (DLCQ) are addressed in parallel with discretized equal time quantization (DETQ) and the fact that Lorentz invariance and microcausality are restored if one can take the continuum limit properly is emphasized. In the free case, it is shown with numerical evidence that the continuum results can be reproduced from DLCQ results for the Pauli-Jordan function and the real part of Feynman propagator. The contributions coming from $k^{+}$near zero region in these cases are found to be very small. In the interacting case, aspects related to the continuum limit of DLCQ results in perturbation theory in momentum space are discussed.
\end{abstract}

Keywords: DLCQ, Microcausality, Pauli-Jordan Function, Continuum limit

*email: asmita@tnp.saha.ernet.in 


\section{Introduction}

The discretized light-cone quantization (DLCQ) [1 - 4] was proposed to study nonperturbative aspects of field theories and is extensively used in practical calculations following Ref. [⿴囗十]. The vacuum is simpler and the treatment of the infrared degrees of freedom is less complicated in this formalism. DLCQ has also been applied in different contexts such as transverse lattice formalism [5] and M-theory [6]. However, at the same time it has some inherent difficulties [7].

In a recent work [8] it is shown that microcausality is violated in the framework of DLCQ. The results of [8] can be summarized as follows. Consider the Pauli-Jordan function in $(1+1)$ dimensional free scalar theory

$$
\Delta(x)=\frac{1}{i}[\phi(x), \phi(0)]=\frac{1}{i} \int \frac{d^{2} k}{2 \pi} \delta\left(k^{2}-m^{2}\right) \epsilon\left(x^{0}\right) e^{-i k \cdot x} .
$$

An explicit evaluation leads to,

$$
\Delta(x)=-\frac{1}{2} \epsilon\left(x^{0}\right) \theta\left(x^{2}\right) J_{0}\left(m \sqrt{x^{2}}\right)=-\frac{1}{4}\left[\epsilon\left(x^{+}\right)+\epsilon\left(x^{-}\right)\right] J_{0}\left(m \sqrt{x^{2}}\right),
$$

where $J_{0}$ is the Bessel function and the light-front variables are defined as $x^{ \pm}=x^{0} \pm x^{1}$. As is evident from Eq. (2), $\Delta(x)$ is zero in the spacelike region due to microcausality. Numerical results show that $\Delta(x)$ is non-vanishing in the space-like region within the box if the box is considered to be lying along light-front longitudinal direction $x^{-}$(i.e., in DLCQ) and hence violates microcausality. Further, the continuum result for $\Delta(x)$ is not restored if one uses DLCQ and let $L \rightarrow \infty$. Similar observation was also made in [9]. This is in contrast to the case when one uses the box to be lying along $x^{1}$ (i.e., in DETQ) where microcausality is not violated within the box as long as $x^{0}<L$ and continuum limit is reproduced.

In view of the fact that DLCQ is already known to produce reasonable results [10], it is necessary to investigate further to clarify what actually is going on. In our opinion, the observation made in Ref. [8] is, at best, superficial as we have clarified below providing the actual picture with numerical evidence.

\section{Comments on Microcausality}

First of all, it should be noted that when one is using DLCQ or DETQ, one is dealing with a theory where the raw output is not the ultimate concern. Only the continuum limit of that output is what we are interested in and presumably can be tested with some experiment. In this sense, it is not our primary concern if some symmetry like boost invariance or even microcausality is violated in the discretized version of the theory. The fact that microcausality may be violated in DLCQ with a fixed box length $2 L$, can be seen trivially once we assume the periodic boundary condition $\phi(L)=\phi(-L)$, since

$$
[\phi(L), \phi(0)]=[\phi(-L), \phi(0)]=[\phi(0), \phi(L)], \quad \Rightarrow \quad[\phi(L), \phi(0)]=0,
$$

for all time. If the continuum result is our guideline, we should have obtained $-\frac{i}{4} \epsilon(L)$ for $x^{+}=0$ and hence the microcausality that is always maintained in the continuum theory is not guaranteed to be respected in DLCQ with fixed L, as also is the case with boost 
invariance. That precisely is the reason for which one has to remove the $L$-dependence of the result obtained from discretized version of the theory (which, in general, may depend on $L$ ) by taking continuum limit to ensure correct physics. Therefore, if the continuum result for Pauli-Jordan function is not reproduced from DLCQ as is claimed in Ref. [8, we are facing a real disaster. In the rest of this work, we show that the situation is not so alarming and try to clarify the intricacy involved in taking the continuum limit, both in DLCQ and DETQ. We also compare and contrast the situations in coordinate space and momentum space.

\section{Comments on $k^{+}=0$ modes}

The Pauli-Jordan function can be given in the momentum representation by integrating $k^{0}$ or $k^{-}$as

$$
\begin{aligned}
\Delta(x) & =-\int_{-\infty}^{\infty} \frac{d k^{1}}{2 \pi \omega_{k}} \sin \left(\omega_{k} x^{0}-k^{1} x^{1}\right) \\
& =-\int_{0}^{\infty} \frac{d k^{+}}{2 \pi k^{+}} \sin \left(k^{-} x^{+} / 2+k^{+} x^{-} / 2\right),
\end{aligned}
$$

where $\omega_{k}=\sqrt{\left(k^{1}\right)^{2}+m^{2}}$ and $k^{-}=m^{2} / k^{+}$.

Notice that the integrand in Eq. (4) is highly oscillatory for $k^{+}$near 0 in contrast to the corresponding equal-time case when $k^{1}$ near 0 as long as the mass is not zero. In general, understanding the role of $k^{+}=0$ modes is very crucial in light-front quantization [7]. The observed discrepancy between DLCQ result and the continuum one for $\Delta(x)$ is generally believed to be due to the elimination of the zero mode $\left(k^{+}=0\right.$, the accumulation point) in DLCQ, at least in the case of free theory [8,9]. To see the actual contribution to $\Delta(x)$, we have performed numerically the integration of Eq. (四) separately for different $k^{+}$regions and the results are shown in Fig. 1. It is clear that the contribution coming from the region where $k^{+}$is very small is negligible compared to that coming from the other region. 
(a)

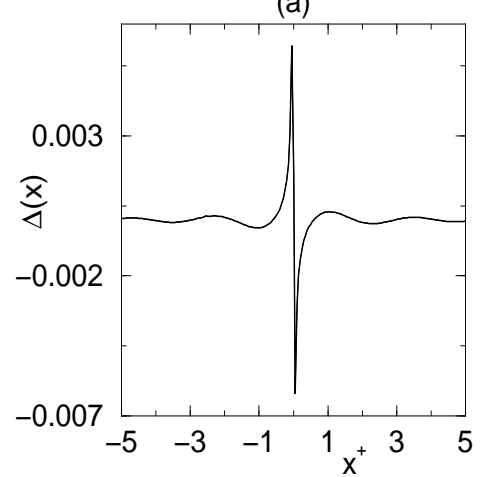

(c)

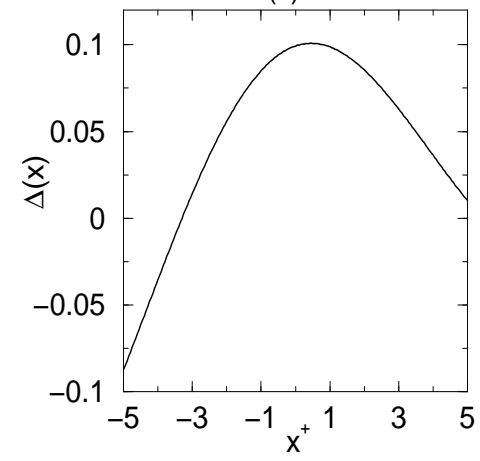

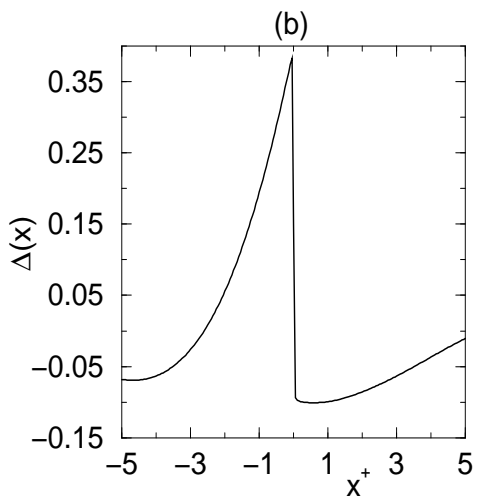

(d)

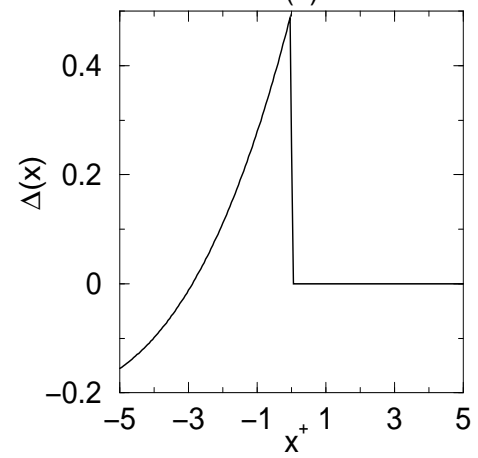

Fig. 1

Fig. 1: Plot of Pauli-Jordan function showing contributions from different regions of $k^{+}$for $x^{-}=-2.0$ and $m^{2}=1$. The ranges of $k^{+}$integrations are (a) $10^{-5}$ to $10^{-3}$, (b) $10^{-3}$ to 1.0 , (c) 1.0 to $10^{3}$ and (d) $10^{-5}$ to $10^{3}$, this curve is the same as that of Eq. (2).

Though it is not possible to reach $k^{+}=0$ numerically, it can be seen that the total result matches with the exact result (Eq. (21)) suggesting that the contribution from the region $k^{+} \leq 10^{-5}$ is even smaller and insignificant. So the discrepancy does not seem to be caused by the zero modes. Our numerical result in the continuum agrees with the assertion in Ref. [7], that zero mode is not an accumulation point for the Pauli-Jordan function. For more discussions on the role of zero modes see Ref. [1, [7].

\section{Continuum Limit}

The discretized versions of $\Delta(x)$ by restricting spatial coordinates, $-L \leq x^{1}, x^{-} \leq L$ and using periodic boundary condition for the field $\phi$ are given by,

$$
\begin{aligned}
& \Delta_{E T}(x)=-\sum_{n=-N}^{N} \frac{1}{2 \omega_{n} L} \sin \left(\omega_{n} x^{0}-n \pi x^{1} / L\right), \\
& \Delta_{L C}(x)=-\sum_{n=1}^{N} \frac{1}{2 \pi n} \sin \left(k_{n}^{-} x^{+} / 2+n \pi x^{-} / L\right),
\end{aligned}
$$

where, in principle, $N \rightarrow \infty$ and for $n$th discrete momentum mode energies are given by $\omega_{n}=\sqrt{n^{2} \pi^{2} / L^{2}+m^{2}}$ and $k_{n}^{-}=m^{2} L / 2 \pi n$ respectively. 
In general, at the end of any calculation in the discretized version of the theory one is expected to get back the continuum result by taking $N \rightarrow \infty$ and $L \rightarrow \infty$ limit. For practical computations, it means that one has to take $N$ sufficiently large for any particular $L$ value such that the summation saturates (in the sense that it does not change appreciably if the value of $N$ is increased). It turns out that if $N / L$ ratio is large then the saturation occurs. In all the results presented $N$ has been taken sufficiently large so that the results are independent of $N$.

Now, in DLCQ in coordinate space, taking the continuum limit is a little tricky. To understand things properly, we perform the summation in Eq. (5) and Eq. (6) numerically and study their behavior by changing the parameters involved, namely, $N, L$ and also the space-time points at which it is calculated.

First, we present the observation made in DETQ. In Fig. 2(a), we have plotted $\Delta_{E T}$ in the continuum theory. From Fig. 2(b), we can clearly see that for a particular $L\left(>x^{0}\right)$ and for fixed $x^{0}, \Delta_{E T}$ actually oscillates about the continuum value and converges to it (at least within the box) as we increase $N$ sufficiently. Since continuum result is reproduced within the box, we see that microcausality is not violated within the box, i.e., yields zero for spacelike region. Outside the box it is a different story altogether, since we just get periodic copies which, of course, differs from the continuum result. Now, increasing only $L$ with $N$ fixed to earlier value, the result again starts oscillating around the continuum value and one could even get widely varying result (non-zero for spacelike region) if $L$ is sufficiently increased as shown in Fig. 2(c) (periodic copies are not shown there).
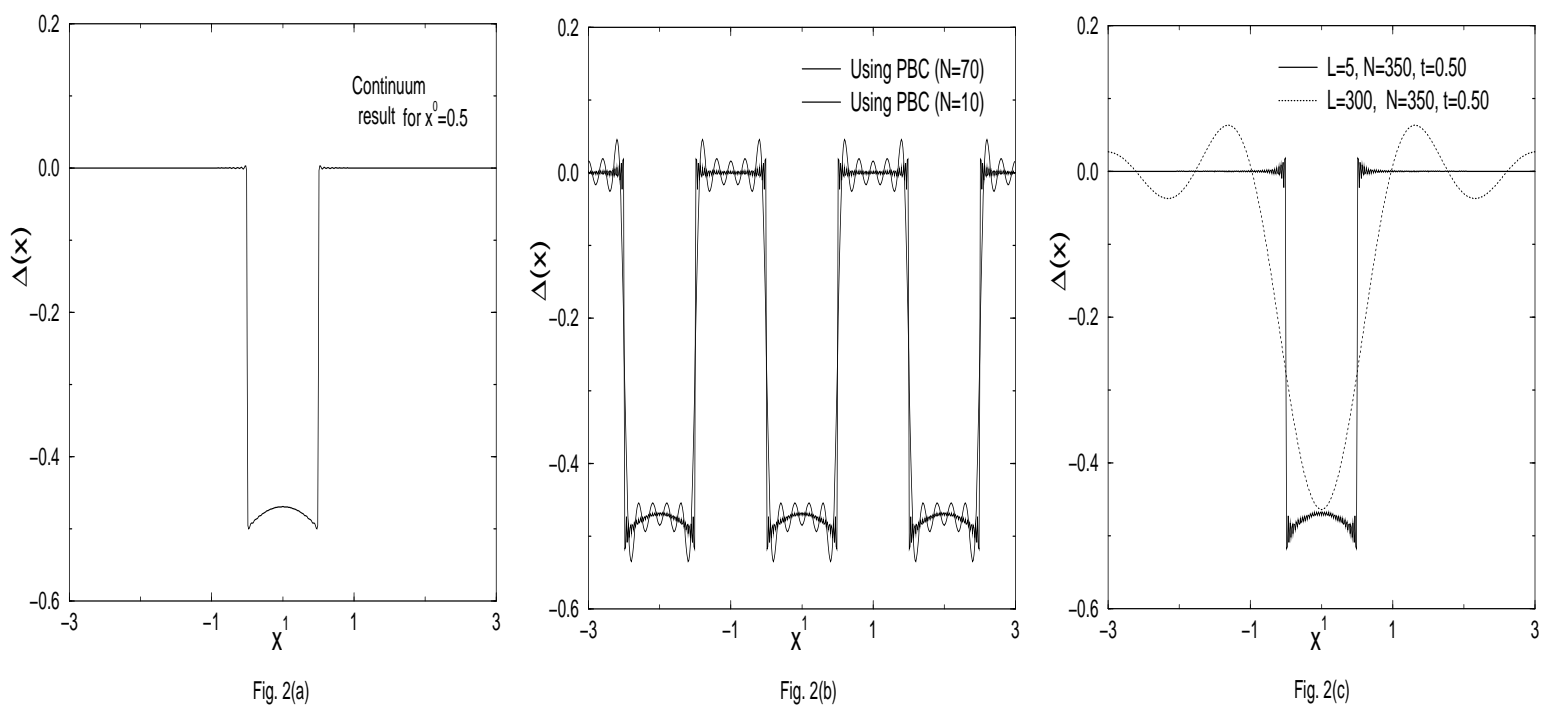

Fig. 2: Plot of Pauli-Jordan function in equal time. 2(a): continuum theory for $x^{0}=0.5$. 2(b): DETQ theory for $L=1$ and two different values of $N$. 2(c): DETQ theory for $N=350$ and two different values of $L$.

This, of course, does not mean that microcausality is violated. It only means that we are far from continuum limit. On the other hand, if one increases $L$ and $N$ simultaneously keeping the ratio $N / L$ large one gets the same continuum result within the box. Thus, 
the effect of taking $L$ to infinity this way simply removes the periodic copies and gives the continuum result everywhere.

These are, of course, very well known results. The main point that we want to drive home here is that if we are somewhat near the continuum limit, then $\Delta_{E T}$ actually oscillates around the exact result and thus, taking an average, one could obtain good agreement with the continuum result. In Fig. 3(a), we have shown the $\Delta_{E T}$ for a particular space-time point as a function of $L$. We see clearly that it fluctuates in a very small range where any value is actually close to the continuum result and hence, the average value in that range is a good approximation for the continuum limit. Further, we notice that as $L$ increases $\Delta_{E T}$ converges slowly but appreciably towards a particular value which is its true continuum limit.

In the case of $\Delta_{L C}$, the situation is different. In particular, for a fixed $L$ the result stabilizes as we increase $N$, but no way near result of the continuum limit within the box, as is also observed in Ref. [8].


Fig. 3(a): Variation of $\Delta(x)$ with $L$ in DETQ, 3(b): Variation of $\Delta(x)$ with $L$ in DLCQ using periodic boundary condition.

Thus, this result on its own is unphysical in the sense that we are unable to test them by some experiment and it is not surprising to observe that microcausality is violated (since it is non-zero in the space-like region). Now, as in Fig. 3(a), we show the similar plot for $\Delta_{L C}$ in Fig. 3(b). We clearly see the striking difference in this case, namely, it varies in a wider range of values and shows hardly any evidence of converging to some value. Nevertheless, it shares one property of $\Delta_{E T}$, namely, it fluctuates around some average value. Taking the clue from earlier plots it suggests that the average value about which it is fluctuating might be a good approximation for the continuum limit for $\Delta_{L C}$. In fact, it turns out to be the case. We have shown this fact in Figs. 4(a), 4(b) and 4(c). Here, at different $x^{+}$and $x^{-}$, we have first calculated these averages for various box length $L\left(>x^{+}, x^{-}\right)$with large $N / L$ ratio and plotted them and the results remarkably agree with the continuum results. Also the discretized result is zero in the spacelike region. The fact that continuum result does not care whether we have imposed periodic or anti-periodic boundary conditions, is also shown in these plots. 
Thus, we see that even though an individual result with fixed $L$ looks unphysical, an assembly of such results for various box length $L$ can be used to extract sensible result in DLCQ and the microcausality, which is required for any sensible physical result, is in no danger here.
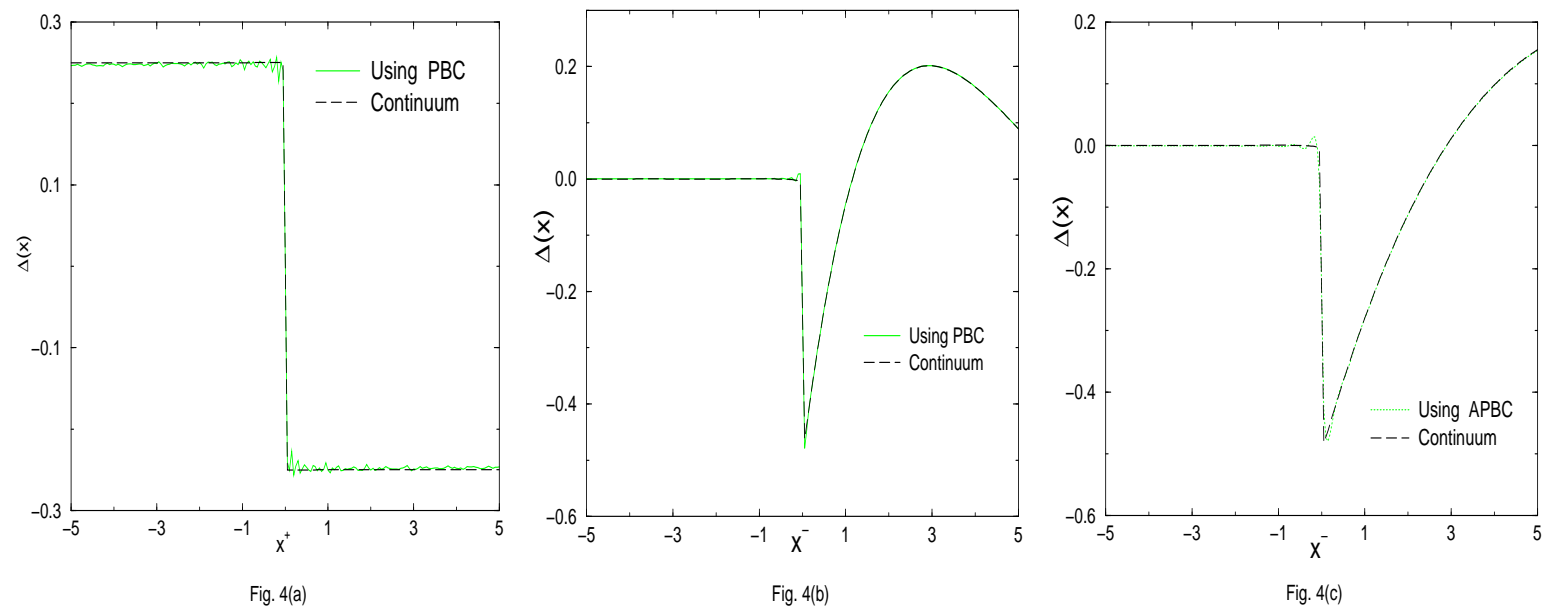

Fig. 4: Plot of $L$ averaged DLCQ result of $\Delta(x)$ using 4(a): periodic boundary condition in comparison with the continuum result for $x^{-}=0.4(\mathrm{~b})$ : periodic boundary condition in comparison with the continuum result for $x^{+}=5.0 .4(\mathrm{c})$ : anti-periodic boundary condition in comparison with the continuum result for $x^{+}=2.0 . \Delta(x)$ is zero in spacelike region in both $4(\mathrm{~b})$ and $4(\mathrm{c})$.

We have also verified that the same feature is evident in Feynman propagator $\Delta_{F}(x)$ as well. Imaginary part of the Feynman propagator being connected to the Pauli-Jordan function $\left(\Delta(x)=2 \epsilon\left(x^{+}\right) \operatorname{Im}\left(i \Delta_{F}(x)\right)\right.$, we simply compare the real part of the propagator in this theory

$$
R e\left(i \Delta_{F}(x)\right)=\frac{1}{8}\left[\epsilon\left(x^{+}\right)+\epsilon\left(x^{-}\right)\right] N_{0}\left(m \sqrt{x^{2}}\right)-\frac{1}{4 \pi}\left[\epsilon\left(x^{+}\right)-\epsilon\left(x^{-}\right)\right] K_{0}\left(m \sqrt{x^{2}}\right)
$$

with the corresponding DLCQ result using anti-periodic boundary condition

$$
R e\left(i \Delta_{F}(x)\right)=\sum_{n=1}^{N} \frac{1}{2 \pi(2 n-1)} \cos \left(\frac{m^{2} L x^{+}}{2(2 n-1) \pi}+\frac{(2 n-1) \pi x^{-}}{2 L}\right) .
$$

In Fig. 5, we have shown the DLCQ result which is obtained by using Eq. (8) for various $L$ and taking averages as we did earlier. It is again in remarkable agreement with the continuum result.

So far we have restricted ourselves to position space. The situation is completely different if calculations are performed in momentum space where the continuum limit is easily achieved as discussed in the next section. This is not surprising as we see that the Fourier transforms of the DLCQ results are the discretized versions of the continuum results. Fourier transform of $\Delta(x)$ for $x^{-}=0$ in DLCQ is given by, 


$$
\Delta\left(k_{j}^{-}\right)=-\frac{1}{2 \pi} \int_{-\infty}^{\infty} d x^{+} e^{-\frac{i}{2} k_{j}^{-} x^{+}} \sum_{n} \frac{1}{2 \pi n} \sin \left(\frac{k_{n}^{-} x^{+}}{2}\right)=-\frac{1}{2 \pi i} \frac{1}{k_{j}^{-}} .
$$

It is the discretized version of the Fourier transform of the continuum result (i.e., $\left.-\frac{1}{4} \epsilon\left(x^{+}\right)\right)$. Similarly, the Fourier transform of $\Delta(x)$ for $x^{+}=0$ in DLCQ is also the discretized version of the Fourier transform of $-\frac{1}{4} \epsilon\left(x^{-}\right)$.

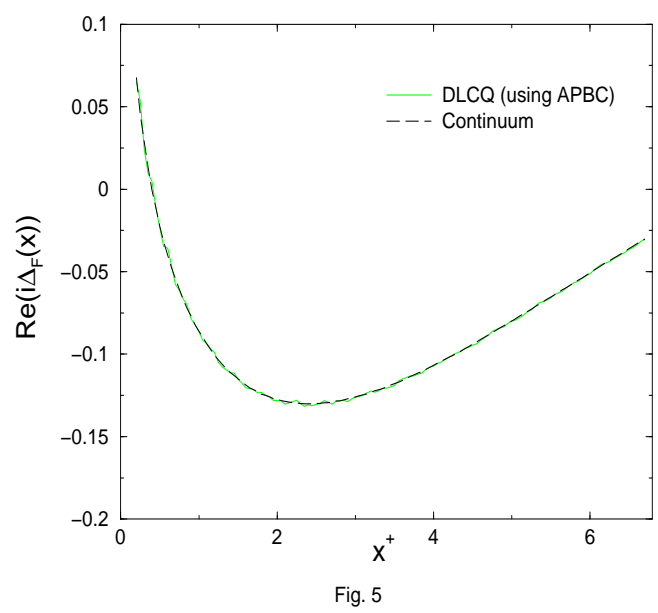

Fig. 5: Plot of $L$ averaged DLCQ result of $\operatorname{Re}\left(i \Delta_{F}(x)\right)$ using anti-periodic boundary condition in comparison with the continuum result for $x^{-}=2.0$.

\section{Interacting Theory}

Till now we have considered Pauli-Jordan function in $(1+1)$ dimensional free scalar field theory and have shown through numerical analysis that one gets the continuum result irrespective of using DETQ or DLCQ. In this section, we investigate the situation by adding a $\phi^{4}$-interaction. In particular, we calculate a scattering amplitude in perturbation theory in DLCQ and compare its continuum limit with the exact result. We shall observe that the continuum limit is obtained much more directly for such entities which are functions in momentum space compared to Pauli-Jordan function, which is a function in position space.

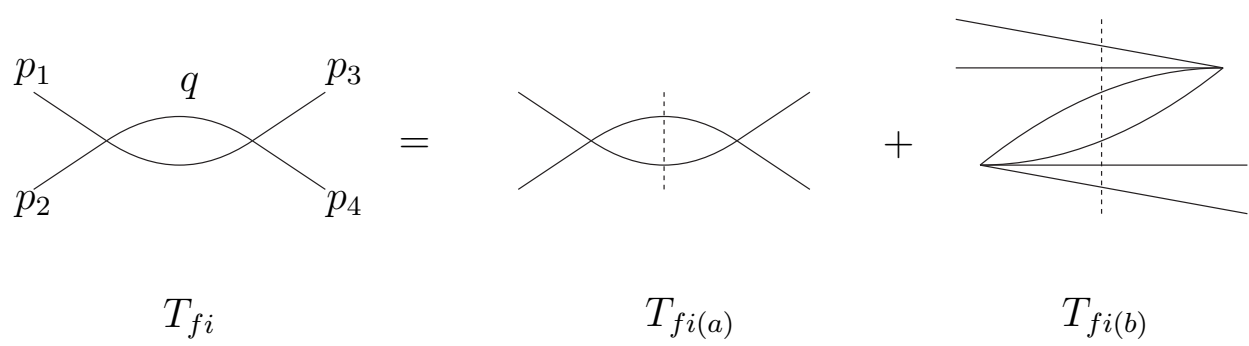

We consider one loop scattering process in $\phi^{4}$ theory. The Feynman diagram shown in the figure is the sum of two time-ordered diagrams. The scattering amplitude of the process is given by, 


$$
T_{f i}=\frac{\lambda^{2}}{8 \pi} \int_{0}^{1} d y \frac{1}{y(1-y) s-m^{2}+i \epsilon}
$$

where $s=\left(p_{1}+p_{2}\right)^{2}$ and $y=\frac{q^{+}}{P^{+}}, P^{+}$is the total longitudinal momentum. We have taken $s<4 m^{2}$. In old-fashioned light-front Hamiltonian perturbation theory, the second diagram $\left(T_{f i(b)}\right)$ in the right hand side is absent. DLCQ result for the first diagram can be easily calculated and is given by

$$
T_{f i(a)}=T_{f i}=\frac{\lambda^{2}}{4 \pi} \sum_{n=1}^{N} \frac{1}{H(2 n-1)[K-(2 n-1)]-m^{2} K+i \epsilon}
$$

We have used the anti-periodic boundary condition here to avoid the zero mode problem which is present in the interacting theory with periodic boundary condition. Here $P^{+}=$ $p_{1}^{+}+p_{2}^{+}=K \pi / L, P^{-}=p_{1}^{-}+p_{2}^{-}=H L / \pi, M^{2}=P^{+} P^{-}=K H$ and $q_{n}=(2 n-1) \pi / L$, with $K$ and $n$ being integer. Also, since internal lines carry momenta which are odd multiple of $\pi / L, K$ should be even and decides the value of $N$, the highest mode in the sum. Note that the result in Eq. (11) is independent of $L$ and the continuum limit is simply obtained by increasing $N$, (i.e., $K$ here) if it stabilizes. It can be easily verified that DLCQ gives the continuum result without any problem as correctly mentioned in Ref. [7]. Here we explicitly show that in perturbative $\phi^{4}$ theory DLCQ in momentum space is free from zero mode problem and at the same time it gives us a comparative picture of DLCQ in position and momentum space.

In old-fashioned equal-time Hamiltonian perturbation theory, contribution comes from both of the time-ordered diagrams. In this case, DEQT result is given by $T_{f i}=T_{f i(a)}+T_{f i(b)}$, where,

$$
\begin{gathered}
T_{f i(a)=} \frac{\lambda^{2}}{16 \pi} \sum_{n=-N}^{N} \frac{1}{\sqrt{n^{2}+\frac{m^{2} L^{2}}{\pi^{2}}}} \frac{1}{\sqrt{(K-n)^{2}+\frac{m^{2} L^{2}}{\pi^{2}}}}\left(\frac{L}{\pi}\right)^{2} . \\
\frac{1}{\left[\sqrt{K^{2}+\frac{s L^{2}}{\pi^{2}}}-\sqrt{n^{2}+\frac{m^{2} L^{2}}{\pi^{2}}}-\sqrt{(K-n)^{2}+\frac{m^{2} L^{2}}{\pi^{2}}}+i \epsilon\right]} \\
T_{f i(b)}=\frac{\lambda^{2}}{16 \pi} \sum_{n=-N}^{N} \frac{1}{\sqrt{n^{2}+\frac{m^{2} L^{2}}{\pi^{2}}}} \frac{1}{\sqrt{(K+n)^{2}+\frac{m^{2} L^{2}}{\pi^{2}}}}\left(\frac{L}{\pi}\right)^{2} \cdot \\
\frac{\left[-\sqrt{K^{2}+\frac{s L^{2}}{\pi^{2}}}-\sqrt{n^{2}+\frac{m^{2} L^{2}}{\pi^{2}}}-\sqrt{(K+n)^{2}+\frac{m^{2} L^{2}}{\pi^{2}}}+i \epsilon\right]}{1}
\end{gathered}
$$

The scattering amplitude in this case depends on $L$. However, when the box length $L$ and the ratio $\frac{N}{L}$ are large the result is independent of $L$ and agrees with the continuum result. The table below compares the results in some arbitrary units as obtained by different formalisms. For DETQ, the calculations are done with $N / L=50$ and $L=5$. But for DLCQ, stability in $K$ depends on the mass, for small mass $K$ should be larger than that for large mass (e.g., for $m^{2}=7, K \geq 15$, for $m^{2}=0.1, K \geq 80$ ). For higher order of accuracy one should take larger values of $N / L$ and $K$. 


\begin{tabular}{|r|c|c|c|c|}
\hline \multicolumn{1}{|c|}{$\mathrm{s}$} & $m^{2}$ & $\frac{T_{f i}}{\lambda^{2}}(\mathrm{DLCQ})$ & $\frac{T_{f i}}{\lambda^{2}}(\mathrm{DETQ})$ & $\frac{T_{f i}}{\lambda^{2}}$ (Continuum) \\
\hline 15.0 & 7.0 & -0.0094 & -0.0094 & -0.0094 \\
\hline 0.2 & 0.1 & -0.6250 & -0.6250 & -0.6250 \\
\hline 3.2 & 1.0 & -0.1101 & -0.1101 & -0.1101 \\
\hline
\end{tabular}

\section{Discussion and Conclusions}

In this work, first, we considered Pauli-Jordan function in $(1+1)$ dimensional free scalar field theory both in equal time and light-front formulations and their corresponding discretized versions. We showed that the microcausality (like the boost invariance) which is required for any sensible theory is not guaranteed to be maintained in the discretized version of the theory. In practice, one is actually interested in the continuum limit of the results obtained in discretized version of the theory, which one can presumably test in some experiment. In general, one expects to obtain the continuum result by taking $N \rightarrow \infty$ and $L \rightarrow \infty$. In DETQ, in coordinate space, for sufficiently large value of $N$ and for any fixed $L$, continuum result is reproduced within the box as long as $x^{0} \leq L$. Thus taking $L \rightarrow \infty$ limit only removes the periodic copies. In contrast, same procedure does not give the continuum result even within the box in DLCQ. Nevertheless, we observe that as a function of $L$, DLCQ result actually fluctuates around the continuum result even when $L$ is very large. We noted the fact that taking an average value from an assembly of such values obtained with various different $L$ reproduces the continuum result with remarkable agreement. We have also obtained the continuum limit of the real part of the propagator starting from DLCQ result. Thus obtaining continuum result ensures microcausality as well as boost invariance.

Note that the Pauli-Jordan function or the Feynman propagator are considered to be the functions in position space here. In contrast, we showed with a specific example that in the perturbative calculations of diagrams in interacting theory where calculations are performed in momentum space, DLCQ result becomes independent of $L$ and the continuum result is obtained simply by increasing $N$ sufficiently. 


\section{REFERENCES}

[1] T. Maskawa and K. Yamawaki, Prog. Theor. Phys. 56, 270 (1976).

[2] A. Casher, Phys. Rev. D14, 452 (1976).

[3] C. B. Thorn, Phys. Rev. D17, 1073 (1978).

[4] H. C. Pauli and S. J. Brodsky, Phys. Rev. D32, 1993, 2001 (1985).

[5] S. Dalley and B. van de Sande, Phys. Rev. D56, 7917 (1997); M.Burkardt and B. Klindworth, Phys. Rev. D55, 1001 (1997); S. Dalley and B. van de Sande, Phys. Rev. Lett. 82, 1088 (1999).

[6] L. Susskind, hep-th/9704080; D. Bigatti and L. Susskind, hep-th/9712072.

[7] S. Tsujimaru and K. Yamawaki, Phys. Rev. D57, 4942 (1998); also see K. Yamawaki, hep-th/9802037.

[8] T. Heinzl, H. Kroger and N. Scheu, hep-th/9908173.

[9] S. Salmons, P. Grange and E. Werner, hep-th/9903101.

[10] For a review, see M. Burkardt, Adv. Nucl. Phys. 23, 1 (1996); S. J. Brodsky, H. C. Pauli and S. S. Pinsky, Phys. Rep. 301, 299 (1998) and references therein. 